\title{
THE DIAGNOSIS OF URINARY TRACT UROLITHIASIS (SMALLANIMALS)
}

\author{
RSTUM ABD-ALAZIZ; ; AHMAD AL-AWAS ${ }^{* *}$ and ADIB AL-ZINA ${ }^{* * *}$ \\ *Veterinarian - Faculty of Veterinary Medicine - AL-Baath University \\ ${ }^{* *}$ Prof in the Faculty of Veterinary Medicine - AL-Baath University \\ *** Lucturer in the Faculty of Veterinary Medicine - AL-Baath University
}

\section{ABSTRACT}

Received: 27/ 9/2012

The study included 99 cats in order to diagnose urinary stones (by the use of radiography and Ultrasonography) Samples were distributed to veterinary clinics in Damascus and faculty of veterinary medicine in HamalSPANAI The results showed three types of urinary stones: where the $(\mathrm{Ca} 0 \mathrm{x})$ is the most common, ollowed by Accepted at: / /2012 struvite stones and Urat according the followed percentage: in cats: $60 \%$ coax, $36 \%$ struvite, $3 \%$ Urat And in the males compered with famales the percentage as followed: 60,6\% males $-36,36 \%$ famales Activity concertration as stones in kidney and bladder according to $\mathrm{x}$-rays and Ultrasonography.

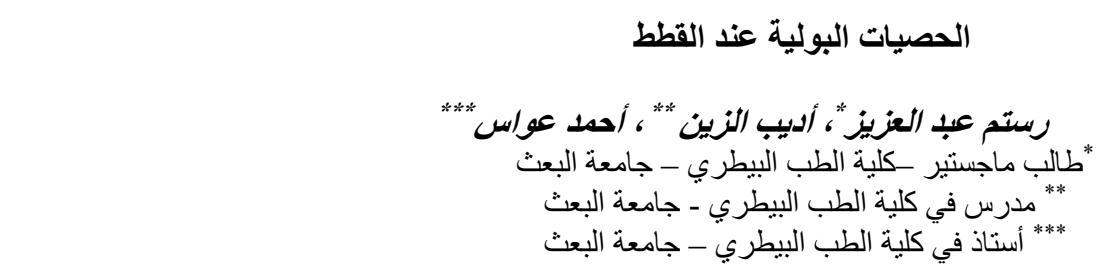

تضدنت الدر اسة فحص 99 قط بهدف تشخيص الحصيات البولية (استخدم التصوير الثعاعي وجهاز الايكوغر افي) كانت العينات موزعة على

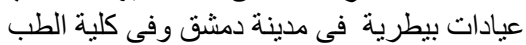

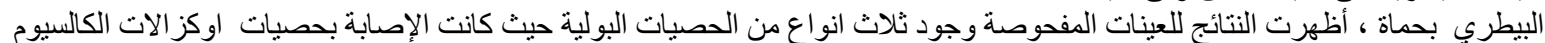

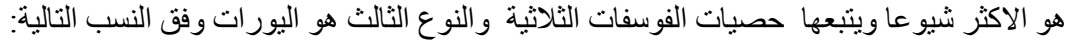

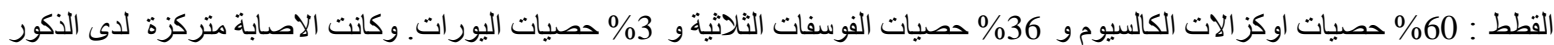

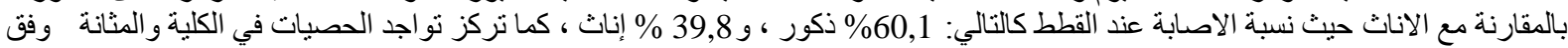
الصور الثعاعية والايكو غر افي.

\begin{abstract}
المقدمة

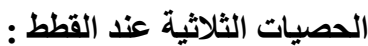

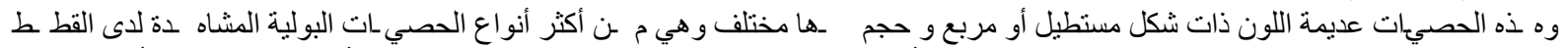

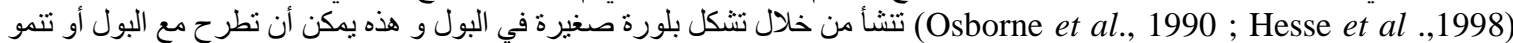

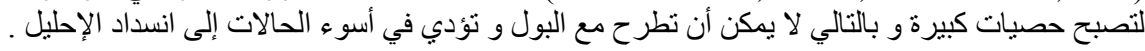

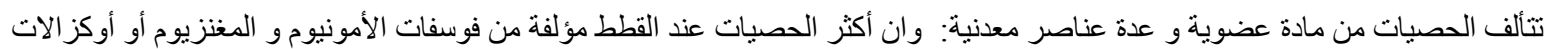

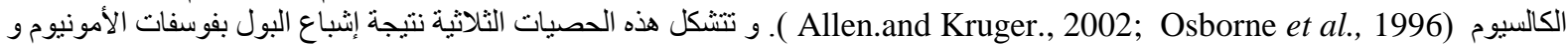

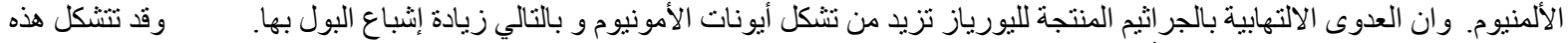

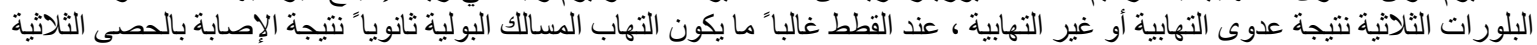

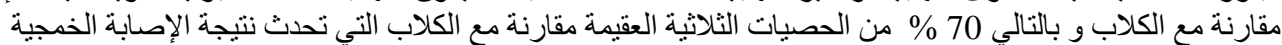
(Leugner, 1988 ; Kraft and Hirschberger ,2000)
\end{abstract} (Feeney and Johnston.,2007; Osborne, et al., 1996)

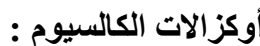

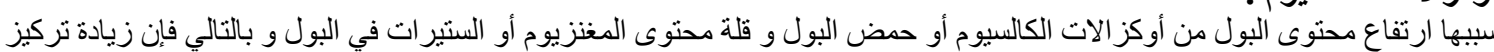

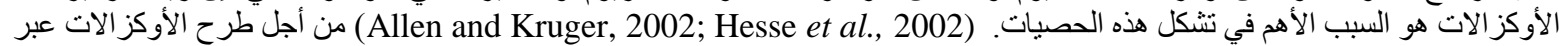

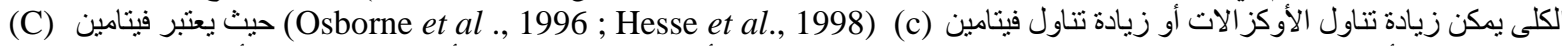

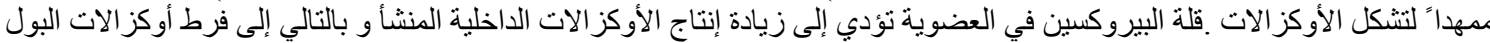

(Osborne et al , 1996 )

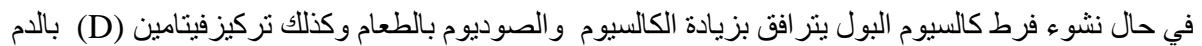
(Osborne et al ., 1996 ; Hesse et al., 1998) هذا و يعتبر خفض قيمة البول وسيلة للوقاية ولمعالجة الحصيات الثلاثية و بنفس الوقت عامل خطورة لتشكل أوكز الات الكالسيوم 


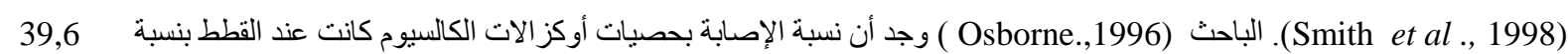
\% أما في عام 1999 أصبحت أكثر من 50 \% \% كما أن القطط المخصية أكثر عرضة للإِصابة بهذه الحصيات و بشكل خاص الذكور تصاب بعدر

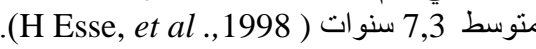

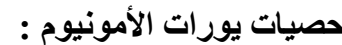

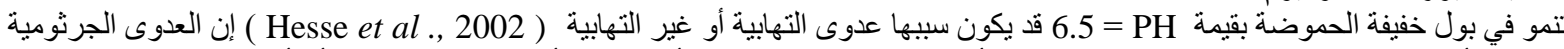

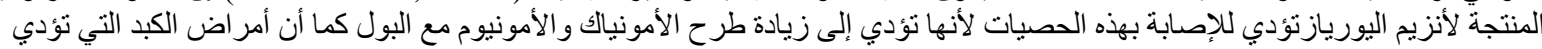

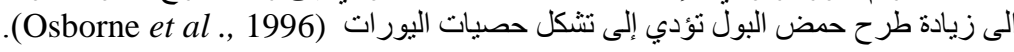
الحيو انات التي تعاني من وجود تحويلة في الوريد البابي مهيئة للإصابة بالحصيات اليورات (Allen and Kruger., 2002) نسبة الإصابة بهات عند القطط 4,8 \% (Osborne , 1996).

تصاب الذكوروالإناث بالمرض وبمتوسط عمر 6,1 سنة (Osborne et al., 1996).

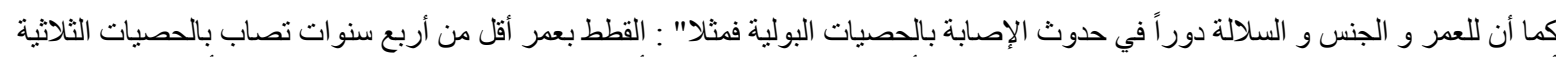

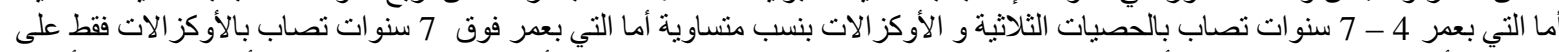

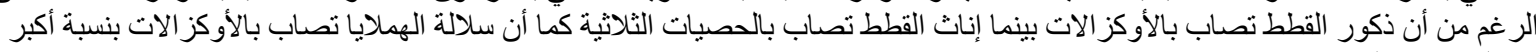
من الحصيات الثلاثية (Hoston et al., 2003).

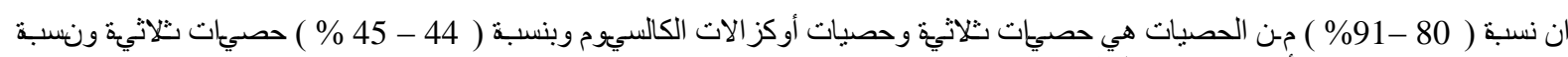
(Hoston et al.

$$
\text { - - تشاف البحث : ت الحصى في المسالك البولية ( التحاليل البيوكيميائية، التصوير الثعاعي ـ التصوير بالايكو). }
$$

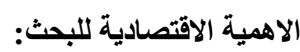

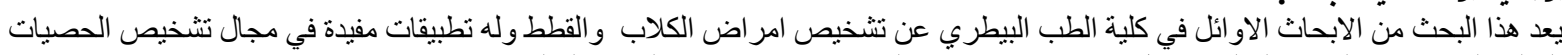

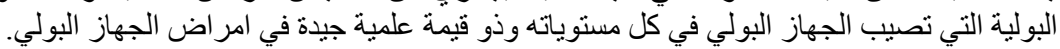

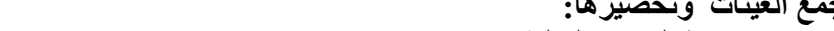

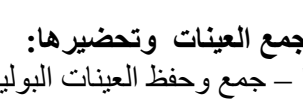

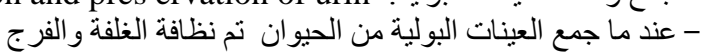
- الجزء الاول من البول الخارج تمن من استبعاده:

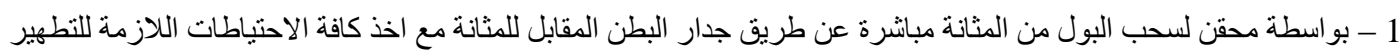

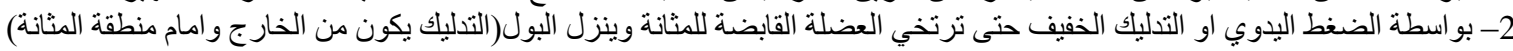

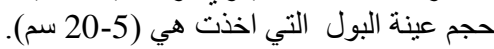
طرق حفظ عينات البول: preservation

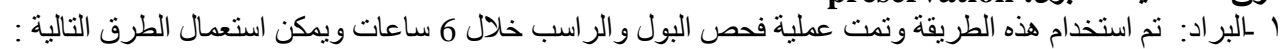

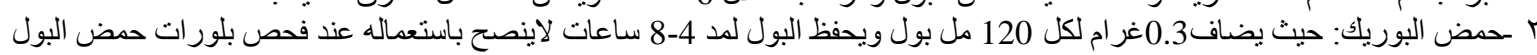

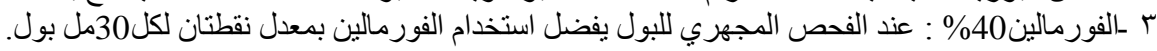

تحليل البول: Urinalysis أ - التحليل الكيميائي للبول:

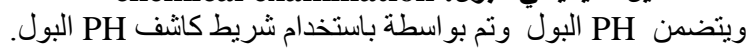

ب - التحليل الفيزيائي للبول: ويتضمن حجم البول ولولونه و الكثافة النو عية وتم باستخدام شريط فاحص البول.

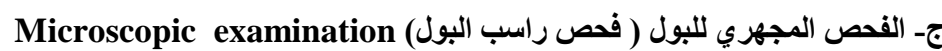

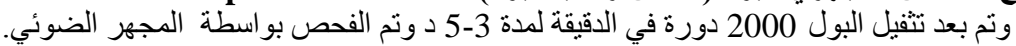

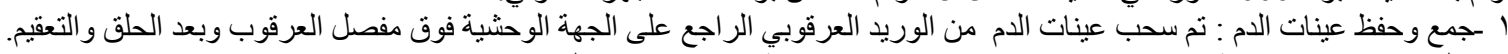

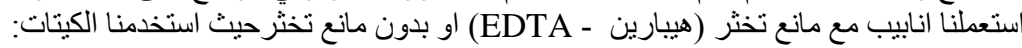
(Sio Systems S.A. Costa Brava 30,Barcelona (Spain) الكيمياء بيوسيستم ونم تثفيل الدم 2000 دورة في الدقيقة لمدة (3-5). التصوير الثعاعي : الأشعة : هي امواج قصيرة غير مرئية كهرومغناطيسية تستخدم في فحص جميع المواد. نفاذية الأشعة تعتمد على كثافة

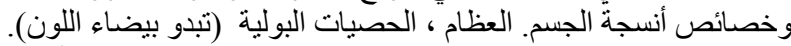
• عند فحص الجهاز البولي اخذنا صورة جانبية وثانية بطنية ظهرية أو ظهرية بطنية لأنية.

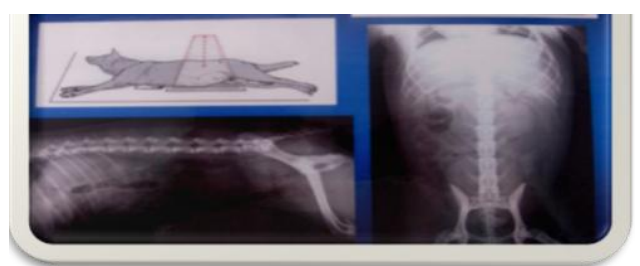




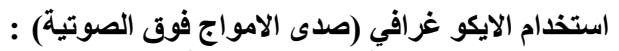

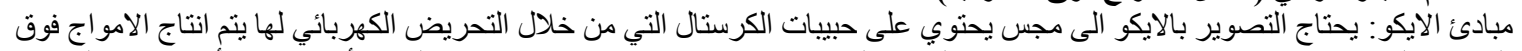

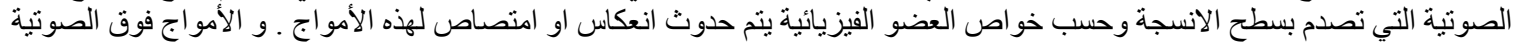

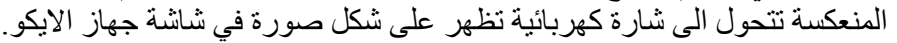

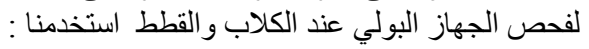

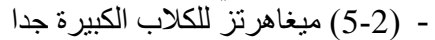

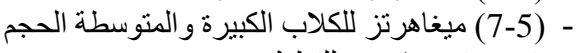
-

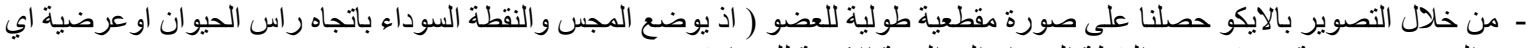
يدور المجس90 درجة بحيث تصبح النقطة السوداء الى الجهة الانسية للحيوان).
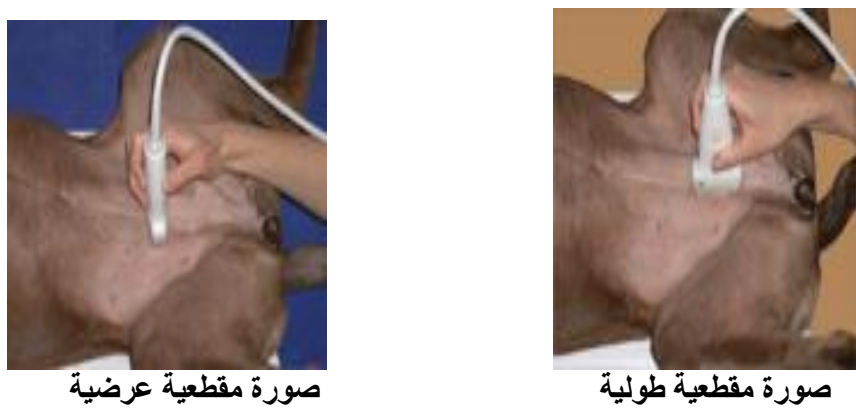

النتائج

\begin{tabular}{|c|c|c|c|c|}
\hline يورات & فوسفات ثلاثية & اوكز الات الكالسيوم & الجنس & السلالة \\
\hline 0 & 7 & 20 & ذكور & هملايا \\
\hline 0 & 9 & 10 & أناث & هملايا \\
\hline 1 & 5 & 14 & ذكور & فارسي \\
\hline 0 & 7 & 5 & أناث & فارسي \\
\hline 1 & 4 & 8 & ذكور & سبامي \\
\hline 1 & 4 & 3 & أناث & سيامي \\
\hline
\end{tabular}

جدول(1) يبين عدد حالات الحصيات المسجلة عند القطط.

\begin{tabular}{|c|c|c|c|}
\hline 2011 & 2010 & 2009 & نوع الحصيات \\
\hline 9 & 24 & 27 & اوكز الات الكالسيوم \\
\hline 6 & 14 & 16 & فوسفات ثلاثية \\
\hline 0 & 1 & 2 & يورات \\
\hline
\end{tabular}

جدول(2) عدد حالات لدى القطط في الفترة بين عام (2009- 2011). مخطط (1) عدد الحصيات البولية المسجلة لدى القطط خلال الاعوام 2009 - 2011

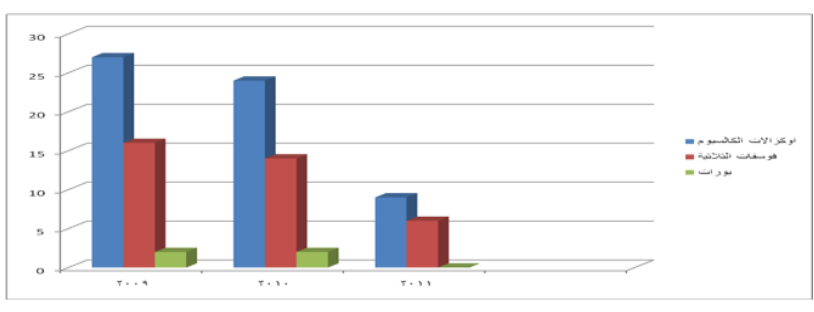

جدول(3) يبين عدد الحالات المسجلة لدى القطط حسب العمر

\begin{tabular}{ccc}
\hline العمر & 2 \\
\hline 3 & 1 & 4 \\
\hline 20 & 20 & 8 \\
\hline 7 & 20 & 10 \\
\hline 6 & 18 & 15 \\
\hline 0 & 1 & 20 \\
\hline 0 & 0 &
\end{tabular}




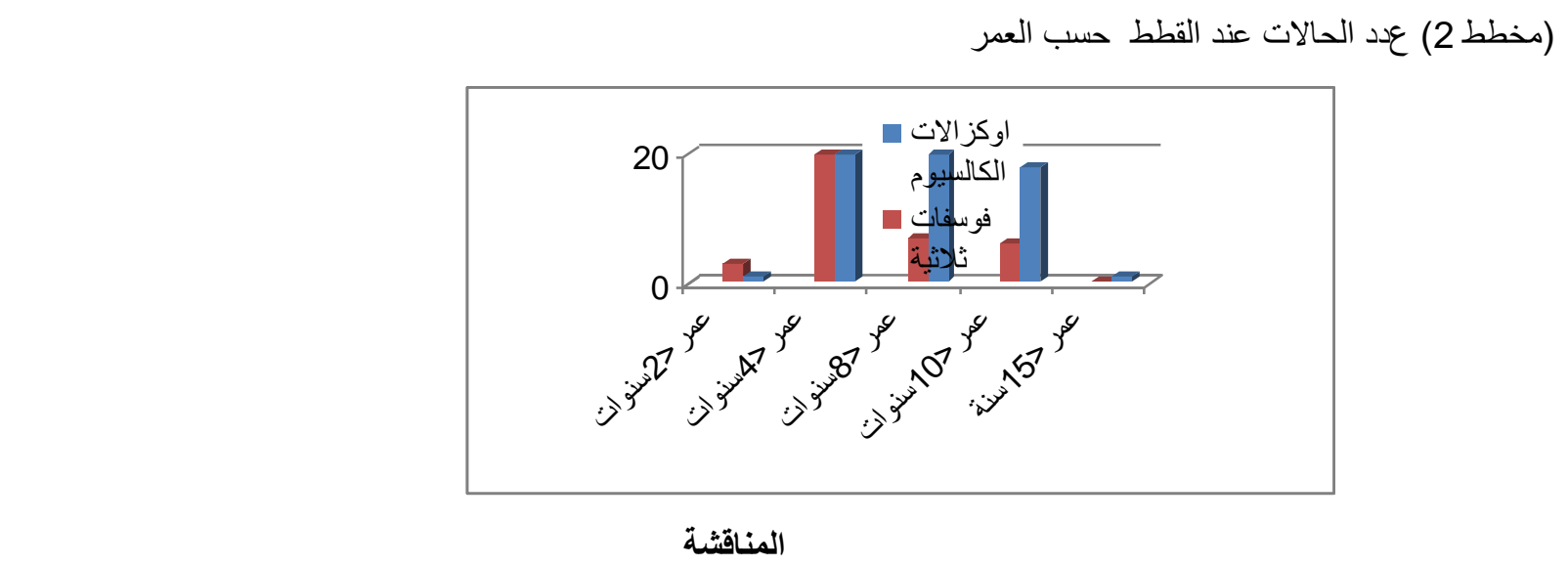

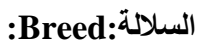

لاى مقارنة الحالات في الجدول (1) لاى القطط نلاحظ مايلي:

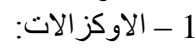

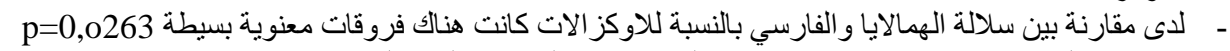

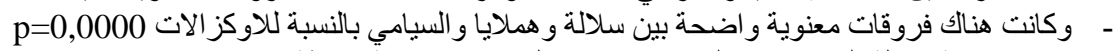

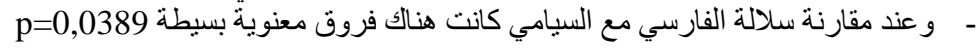

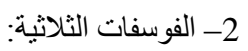

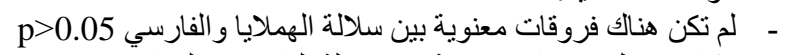

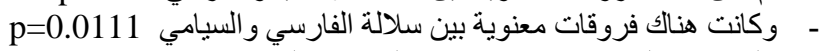
- م- ولم تكن هناك فروقات معنوية بين الهمالايا والسيامي

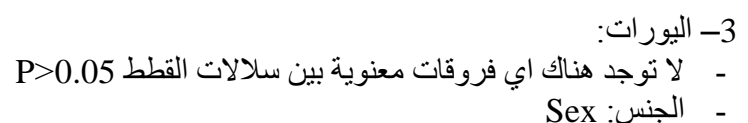

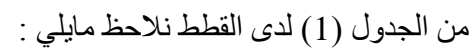

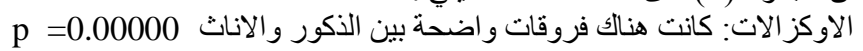

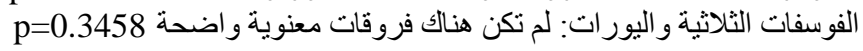

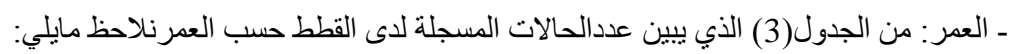

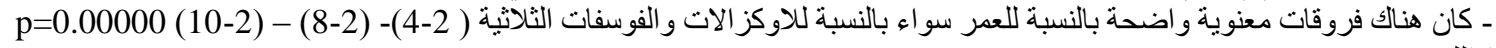

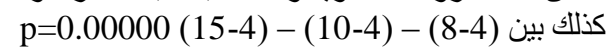

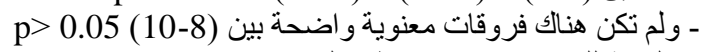

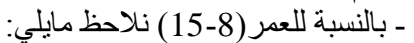

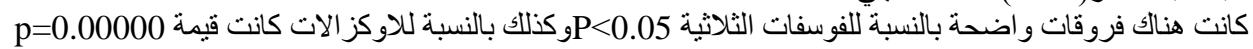

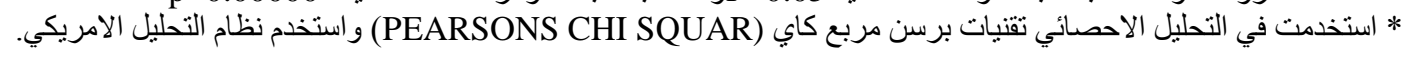
جدول( 4) يبين نتائج الفحوصات المخبرية لدى القطط التي سجل لديها الاصابة بحصيات الفوسفات الثلاثية (المتوسط الحسابي والانحر اف

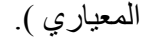

\begin{tabular}{|c|c|c|c|c|}
\hline $\begin{array}{l}\text { CREATININE } \\
\mathrm{mg} / \mathrm{dl}\end{array}$ & $\begin{array}{c}\text { URIC ACID } \\
\mathrm{mg} / \mathrm{dl}\end{array}$ & $\begin{array}{l}\text { UREA } \\
\mathrm{mg} / \mathrm{dl}\end{array}$ & $\begin{array}{c}\text { SPECIFIC qravity } \\
\text { PH }\end{array}$ & عدم وجود رواسب =0 \\
\hline Median $=0.86$ & 3.1 & 21 & $\begin{array}{c}1.02 \\
8\end{array}$ & 0 \\
\hline $\operatorname{Std}=0.08$ & 0.54 & 2.45 & $\begin{array}{c}0.02 \\
0.1\end{array}$ & 0.35 \\
\hline
\end{tabular}

جدول (5) يبين نتائج الفحوص المخبرية لاى القطط الني سجل لديها اليورات.

\begin{tabular}{|c|c|c|c|c|c|}
\hline $\begin{array}{l}\text { CREATININE } \\
\mathrm{mg} / \mathrm{dl}\end{array}$ & $\begin{array}{l}\text { URIC ACID } \\
\mathrm{mg} / \mathrm{dl}\end{array}$ & $\begin{array}{l}\text { UREA } \\
\mathrm{mg} / \mathrm{dl}\end{array}$ & SPECIFIC qravity & $\mathrm{PH}$ & $\begin{array}{c}\text { Unorganized sedliment } \\
1 \text { وجود رواسب=1 }\end{array}$ \\
\hline$M$ edian $=1.4$ & 6.3 & 35 & 1.02 & 5 & 1 \\
\hline Std $=0.07$ & 0.2 & 1.73 & 0 & 0 & 0 \\
\hline
\end{tabular}


$\underline{\text { Assiut Vet. Med. J. Vol. } 58 \text { No. } 135 \text { October } 2012}$

جدول (6) يبين نتائج الفحوص المخبرية لدى القطط التي سجل لديها لاصابة حصيات اوكز الات الكالسيوم.

\begin{tabular}{|c|c|c|c|c|c|}
\hline $\begin{array}{c}\text { CREATININE } \\
\mathrm{mg} / \mathrm{dl}\end{array}$ & $\begin{array}{c}\text { URIC ACID } \\
\mathrm{mg} / \mathrm{dl}\end{array}$ & $\begin{array}{c}\text { UREA } \\
m g / d l \\
\text { SPECIFIC qravity }\end{array}$ & $\mathrm{Ph}$ & وجود رو اسب=1 & $\begin{array}{c}\mathrm{CA} \\
\mathrm{mg} / \mathrm{dl}\end{array}$ \\
\hline Median 1.0125 & 5 & $\begin{array}{c}30 \\
.1 .02\end{array}$ & 55 & 1 & 9 \\
\hline $\mathrm{S} \mathrm{td}=0.25$ & 1.479 & $\begin{array}{c}3.218 \\
0.0048\end{array}$ & 0.3 & 0.4 & 0.3 \\
\hline
\end{tabular}

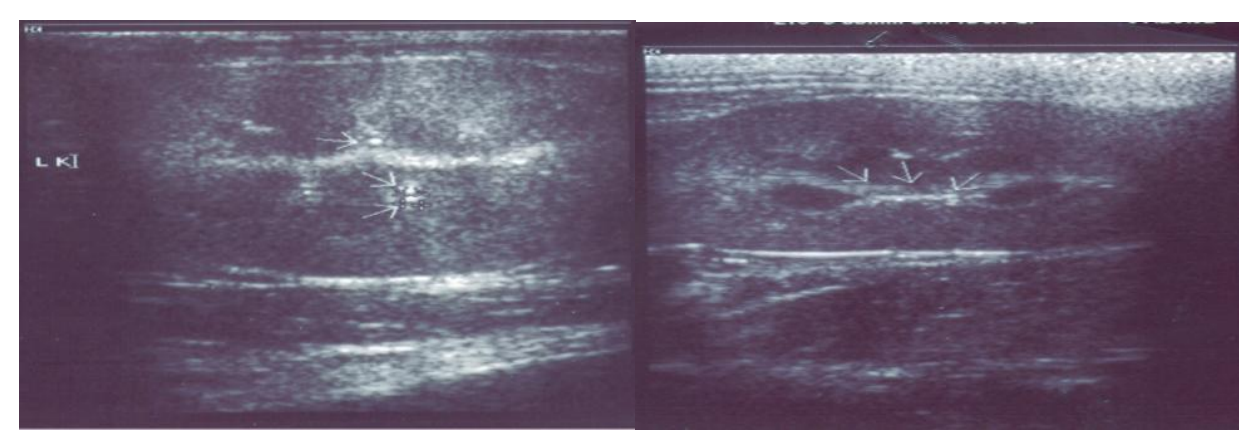

حصيات الكلية

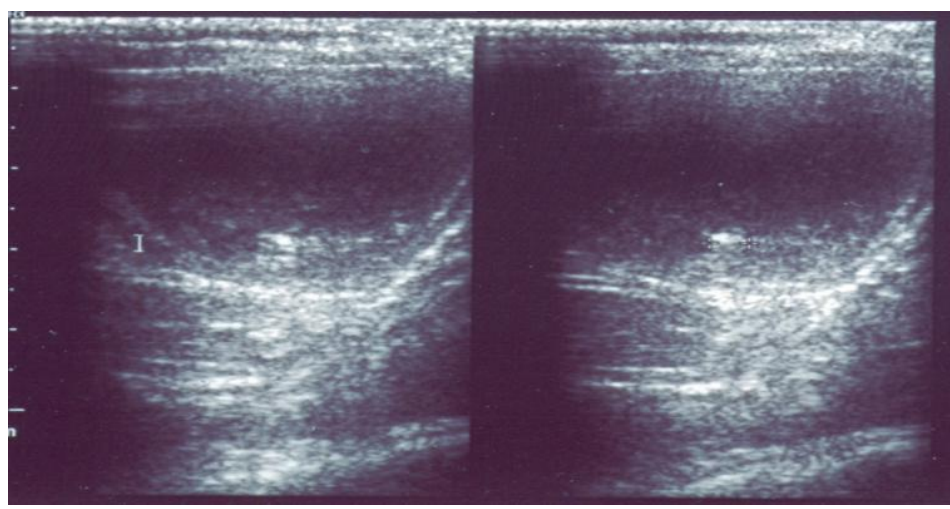

حصيات المثانة

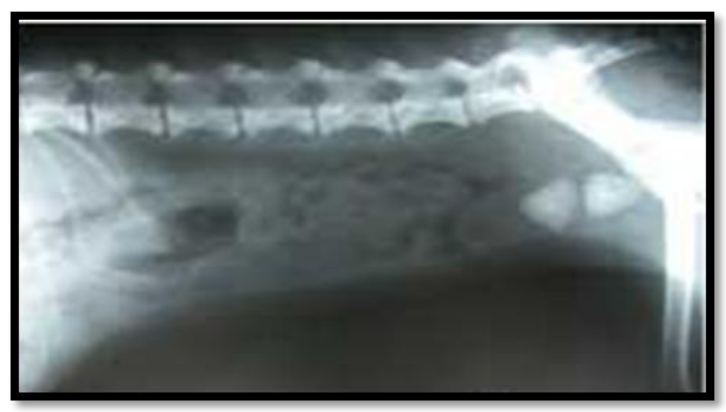

صورة شعاعية لحصيات في المثاتة

مناقشة النتائج: تبين من خلال الدر اسة كما يوضحه الجدول رقم (1) ان القطط تصاب بشكل رئيسي بثلاث انو الناع من الحصيات:

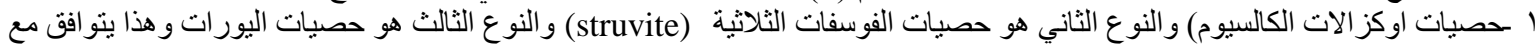
إحدى الدر اسات.

$$
\text { ان أكثر الحصيات عند القطط مؤلفة من فوسفات الأمونيوم و المغنزيوم أو أوكز الات الكالسيوم }
$$
.(Allen and Kruger., 2002; Osborne et al ., 1996)

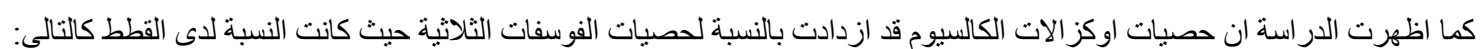

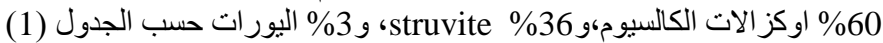

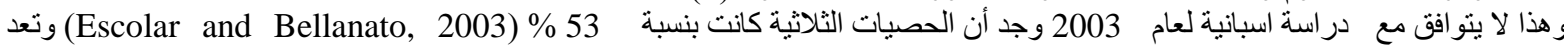
الحصيات الثلاثية الأكثر مشاهدة عند القطط. (Lekcharoensuk et al ., 2000 ; Thumchou et al ., 1996). 


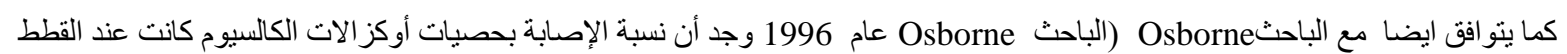
بنسبة 39,6 \% م أما في عام 1999 أصبحت أبثر من 50 \%

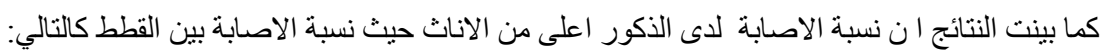

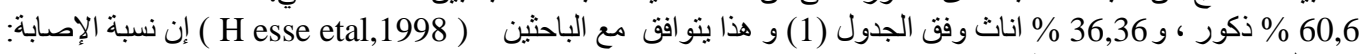

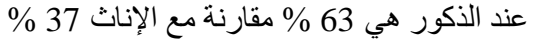

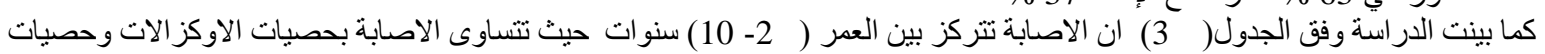

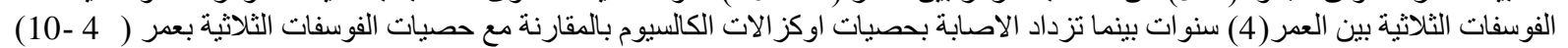

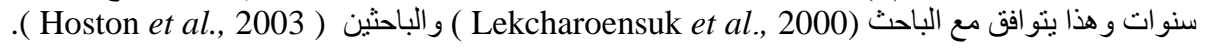
الحصية الثالثة الأكثر شيو عا في القطط هي اليورات.

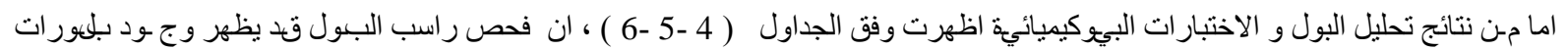

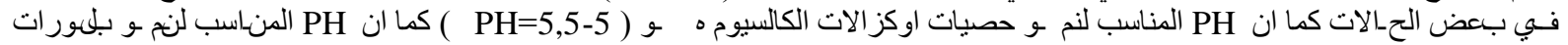

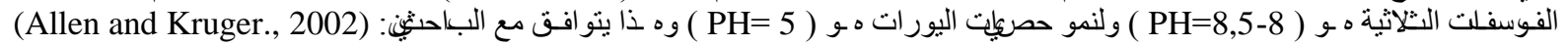

.( Pastoor et al ., 1994 ; Lekcharoensuk et al .,2001)

كما ان معظم حـالات الفوسفلت الثناثية قد ثو افقت م-ع التهابات المساللك البولية وه ذا يتو افق مع البلحثين : (Osborne et al., 1996 ; Ling et al.,1990)

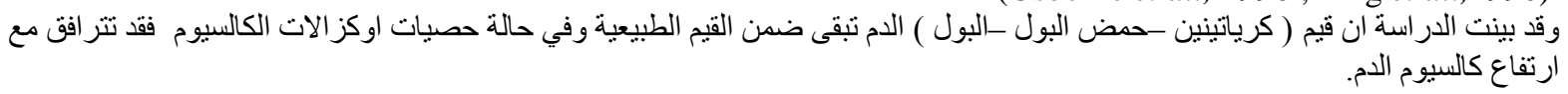

وقد تم تاكيد تشخيص الحصيات بو اسطة بو اسطة الصور الشعاعية وبو اسطة استخدام جهاز الايكو غر افي حيث بينت الصور انها تتوضع بشكل

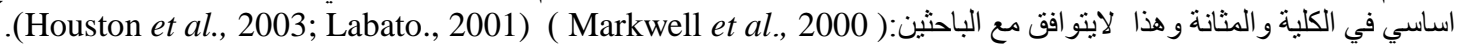

(استتناجات: الاستي

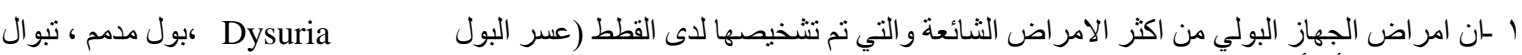
أجulsurie

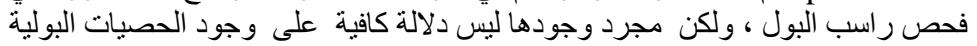

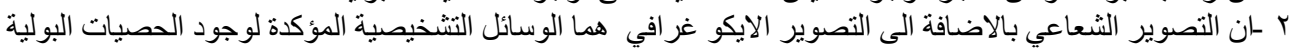

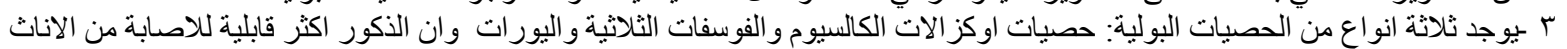

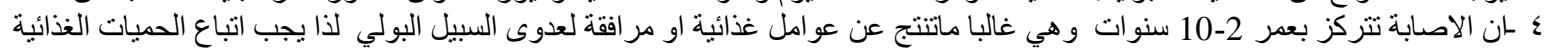
المناسبة و المعالجة السريعة لعدوى السبيل البولي. التوصيات: 1 ـ الاعتماد في التغذية على الحمية الغذائية المناسبة و عدم الاعتماد في التغذية على الاغذية الجافة بل الاغذية الرطبة

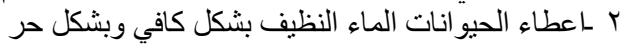

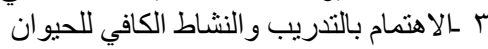

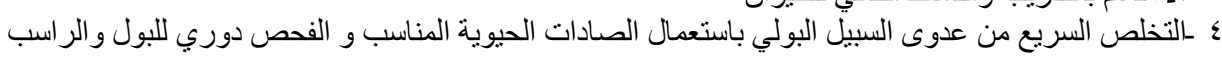

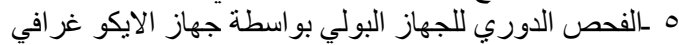

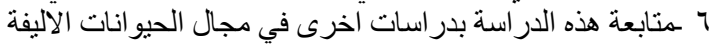

\section{المراج-ع \\ REFERENCE}

Allen, T.A. and Kruger, J.M. (2002): Erkrankungen der unteren Harnwege bei Katzen In: Hand M.S.,Thatcher C.D., RemillarR.L., RoudebushP. Hrsg. Klinische Diätetik für Kleintiere, Band 2 Schlitersche Verlag, Hannover.

Escolar, E. and Bellanato, J. (2003): Analysis of feline urinary calculi and urethral plugs by infrared spectroscopyand scanning electron microscopy Vet. R. $152: 625-628$.

Feeney, D.A.; Johnston, G.R. 2007: The kidnes and ureters. In : Th rall DE, ed .Textbook of veterinary Diagnostic Radiology ., 693-707.

Hesse, A.; Steffes, H. J. and Graf, C. (1998): Pathogenetic factors of urinary stone formation in animals J. Anim. Physiol. a. Anim. Nutr. 80: 108-119.

Hesse, A.; Tiselius, H.-G. and Jahnen, A. (2002): Urinary Stones Karger Verlag, Basel.

Houston, D.M.; Moore, A.E.P.; Favrin, M.G. and Hoff, B. (2003): Feline urethral plugs and bladder uroliths: A review of 5484 submissions 1998- 2003 Can. Vet. J. 44: 974-977.

Kraft, W. and Hirschberger, J. (2000): Kleintierkrankheiten Band 1: Innere Medizin Verlag Eugen Ulmer, Stuttgart8 Literaturverzeichnis 142.

Labato, M. A. (2001): Mamaging urolithiasis in cats.Veterinary Medicine: $708-718$.

Lekcharoensuk, C.; Lulich, J. P.; Osborne, C. A.; Koehler, L. A.; Urlich, L. K.; Carpenter, K. A. and Swanson, L. L. (2000): Association between patient-related factors and risk of calcium oxalate and magnesium ammonium phosphate urolithiasis in cats. J Am Vet Med Assoc 217: 520 - 525 v. Literaturverzeichnis 147. 
Lekcharoensuk, C.; Osborne, C.A.; Lulich, J.P.; Pusoonthornthum, R.; Kirk,C.A.; Ulrich, L.K.; Koehler, L.A.; Carpenter, K.A. and Swanson, L.L. (2001): Association between dietary factors and calcium oxalate and magnesium ammonium phosphate urolithiasis in cats J. Am. Vet. Med. A. 219 (9): 1228-1237.

Leugner, S. (1988): Die Ernährung beim felinen urologischen Syndrom (F.U.S.) Wien. tierärztl. Mschr. 3: 105 112. 8 Literaturverzeichnis 143.

Ling, G. V.; Franti, C. E.; Ruby, A. L. and Johnson, D. L. (1990): Epizootiologic evaluation and quantitative analysis of urinary calculi from 150 cats. JAVMA 196: 1459 - 1462.

Markwell, P. J.; Robertson, W. G.; Stevenson, A. E. (2000): Urolithiasis: a comparison of humans, dogs and cats. In: Rodgers AL, Hibbert BE, Hess B, Khan SR, Preminger GM (eds) Urolithiasis 2000, Vol. 2. University of Cape Town Rondebosch. pp $785-788$.

Osborne, C.A.; Lulich, J.P.; Kruger, J.M.; Polzin, D.J.; Johnston, G.R. and Kroll, R.A. (1990): Medical dissolution of feline struvite Urocystoliths J. Am. Vet. Med. A. 196 (7): 1053-1063.

Osborne, C.A.; Lulich, J.P.; Thumchai, R.; Bartges, J.W.; Sanderson, S.L.; Ulrich, L.K.; Koehler, L.A.; Bird, K.A. and Swanson, L.L. (1996): Diagnosis, medical treatment, and prognosis of feline urolithiasis Vet. Cl. N. Am.: Sm. Anim. Pract. 26 (3): 589-627.

Pastoor, F.J.H.; Opitz, R.; Van't Klooster, A.Th. and Beynen, A.C. (1994): Substitution of dietary calcium chloride for calciu carbonate reduces urinary $\mathrm{pH}$ and urinary phosphorus excretion in adult cats Vet. Q. 16 (3): 157-160.

Smith, B. H. E.; Stevenson, A. E. and Markwell, P. J. (1998): Urinary relative supersaturations of calcium oxalate and struvite in cats are influenced by diet. J Nutr 128: $2763-2764$.

Thumchai, R.; Lulich, J.; Osborne, C.A.; King, V.L.; Lund, E.M.; Marsh, W.E.; Ulrich, L.K.; Koehler, L.A. and Bird, K.A. (1996): Epizootiologic evaluation of urolithiasis in cats: 3498 case (1982-1992) J. Am. Vet. Med. A. 208 (4): 547-551. 